\title{
A Semiautomated Approach for Quality Controlling Large Historical Ocean Temperature Archives
}

\author{
Ann Gronell and Susan E. WijfFels \\ CSIRO Marine and Atmospheric Research, Hobart, Tasmania, Australia
}

(Manuscript received 27 October 2006, in final form 22 June 2007)

\begin{abstract}
This paper describes a method consisting of both automated statistical screening and manual quality control through expert visual inspection, which produces a historical ocean temperature archive of high quality - that is, nearly all profiles are unique (duplicate elimination) and $95 \%$ of bad data is eliminated. The complete process involves comprehensive duplicate elimination, an unreasonable gradient check, and statistical screening to distill out suspect profiles, which are then only eliminated (or partially so) during an expert manual visual inspection step. Statistical screening was optimized using an archive of known quality. Two iterations of statistical screening were required to identify the bulk of the bad data. Of an archive of about 121000 profiles, the authors found they had to manually inspect $35 \%$ of profiles to remove $95 \%$ of the bad data. While costly, they argue such an effort is worthwhile so that the historical ocean temperature archives, which have cost the global community millions of dollars to obtain, are made more immediately useful for climate and ocean sciences. An archive of upper ocean temperature profiles from the Indian Ocean is near completion and extensions into the Pacific Ocean have begun.
\end{abstract}

\section{Introduction}

As the appreciation of the role of the ocean in interannual and decadal climate variability grows, the need to map past and sometimes subtle ocean changes increases. Historical ocean datasets are being compiled through the highly successful Global Oceanographic Data Archeology and Rescue project (Levitus et al. 1994, 2005) and other international programs such as the United Nations Educational, Scientific, and Cultural Organization's (UNESCO) International Ocean Data Exchange program. However, the resulting archives often contain many duplicates of the same profile as well as data of varying quality. Because the archives are so large (millions of profiles), hand quality controlling every profile is not affordable. Here we describe methods that use a combination of expert manual quality control techniques and automated statistical tests that allow the quality of these historical archives to be dramatically increased, thereby increasing the value of these important datasets for climate and ocean studies and reanalysis efforts.

Corresponding author address: Ann Gronell, CSIRO Marine and Atmospheric Research, Hobart, TAS 7000, Australia.

E-mail: ann.thresher@csiro.au
As part of the Commonwealth Scientific and Industrial Research Organization's (CSIRO's) division of Marine and Atmospheric Research (hereafter CMAR) development of a network of repeat expendable bathythermograph (XBT) lines from ships of opportunity (SOOP; see Meyers and Pigot 2000), a scientific quality control system for upper-ocean temperature data was developed (see Gronell 2002). In this system, a qualified operator examines each profile and flags any faults to ensure that "bad" data do not enter the final archive and thus products. Flags are also applied to features that allow us to map such things as temperature inversions and eddy/front systems. This system is documented in the CSIRO Quality Control Cookbook (Bailey et al. 1994); the program that implements these quality control decisions is called Matlab-based Quality Evaluation of Subsurface Temperature Data (MQUEST).

While the temperature observing system implemented in 1983 continues to deliver routine repeat data along fixed lines, studying variability before this time proved difficult due to the mixed quality found in assembled historical data. It was obvious that we required a method to "clean up" this data, but it soon became clear that this is no simple task. The historical dataset is large, with approximately 400000 profiles in the Indian 
Ocean alone and around seven million worldwide. To quality control (hereafter QC) these data to the standard we require would take many years if each profile were to be examined individually. We therefore sought a way to discriminate between profiles that contained "good" and those that contained "suspect" data-a socalled semiautomated system that would involve an automated process to select those profiles that required a closer look using our MQUEST software. "Bad" data could then be flagged by an operator to effectively remove it from the final archive. We undertook a pilot project, which is reported here, to determine whether the increase in data quality would be worth the effort required. The southeast Indian Ocean from latitudes $70^{\circ} \mathrm{S}-0^{\circ}$ and longitudes $90^{\circ}-145^{\circ} \mathrm{E}$ was chosen as a test region for which a method would be developed and tested.

We refer to the resulting quality control system and related archive as the Quality-controlled Ocean Temperature Archive (QuOTA). At present, the method is being applied to the entire Indian Ocean to produce a new higher-quality Indian Ocean thermal archive for climate studies. This archive will be available online when finished. Below we detail the procedures developed, and then compare the costs and results with other statistical methods used previously.

\section{Methods}

The QuOTA system consists of several steps, beginning with data assembly, elimination of duplicate profiles (which occurs when datasets from multiple sources are combined), checking for unreasonable gradients, statistical screening against climatology, and finally, manual quality control of suspect profiles. Below we describe each step in detail.

\section{a. Step 1: Data assembly/formats}

For construction of our initial test dataset, we chose to use as our master dataset all data that had passed through our scientific quality control processes. These included all profiles from the CSIRO Ship-ofOpportunity program and the World Ocean Circulation Experiment (WOCE) Thermal Data Archive. Each of these profiles had been individually checked and all data flagged either as good or bad. To these basic datasets, we added data from several other large data assemblies. These included more recent data from the Australian Bureau of Meteorology that have also been fully quality-controlled, observed level data from the 2001 World Ocean Atlas (hereafter WOA 2001; Stephens et al. 2002), and 1964-96 data from the Far
Seas Fisheries Laboratory (T. Watanabe 2000, personal communication). We also included some data from early CSIRO research cruises and the Insititut de Recherche pour le Developpement, Noumea, New Caledonia. The data were collected from 1826 to 2003 though $94 \%$ of the data were collected since 1960 .

The archive, then, consisted of data from mixed instruments: Nansen casts, mechanical bathythermographs (MBTs), XBTs, conductivity-temperaturedepth recorders (CTDs), and various compressed/ truncated versions of these profiles that may have passed through the global telecommunications system (GTS) and other pathways before entering the national and international data archives. Often, a profile may first arrive in the archives in a low vertical resolution and low precision version via the real-time GTS, and then another higher-quality and full precision copy will arrive several years later. Such duplicates are highly problematic for some scientific analyses.

XBTs that have come from widely different sources presented a problem because profiles collected before 1995 used different coefficients in the equation to calculate depth than did those post-1995. This resulted in a depth error of approximately $3 \%$. In addition, not everyone changed their methods at the same time. So profiles with uncorrected depths were included in data from some of our sources. In coordination with the National Oceanographic Data Center (NODC; C. Sun 1996, personal communication), we developed routines to find and correct these profiles. This program was run over the data before any further steps were taken, ensuring that all profiles were measured to the same depth accuracy.

We have chosen to store our database in a netCDF version of MEDS-ASCII (see http://www.nodc.noaa. gov/GTSPP/document/datafmt/medsfmt.html). This format is similar to the WOCE netCDF format distributed worldwide with one major difference. We allow multiple data types within each file; that is, temperature, salinity, and conductivity are all contained in a single file if they were collected simultaneously. In our usage, a "station" can then contain more than one "profile." Each station is held in a single file, accessed by information in a "keys" file that holds metadata for all the stations in the database. This netCDF file contains a unique identifying number for each station along with the date-time, latitude-longitude, and ship call sign or other identifiers if present. We are only considering the QC of temperature so all tests are carried out on the temperature profile within a station file.

We keep two versions of each station: a raw, unchanged version that is never edited, and a version that contains extensive history records documenting any 
TABLE 1. Data quality classes.

\begin{tabular}{|c|c|c|}
\hline Class & Quality & Description \\
\hline Flag 0 & No QC done & $\begin{array}{l}\text { Flag } 0 \text { data are the level at which all data enter the working archive. They have not yet been } \\
\text { quality controlled. }\end{array}$ \\
\hline Flag 1 & Good data & $\begin{array}{l}\text { Flag } 1 \text { data are top-quality data in which no malfunctions have been identified and all real } \\
\text { features have been verified during the quality control process. }\end{array}$ \\
\hline Flag 2 & "Probably" good data & $\begin{array}{l}\text { Flag } 2 \text { data are good data in which some features (probably real) are present but these are } \\
\text { unconfirmed. Class } 2 \text { data are also data in which minor malfunctions may be present but } \\
\text { these errors are small and/or can be successfully corrected without seriously affecting the } \\
\text { overall quality of the data. }\end{array}$ \\
\hline Flag 3 & "Probably" bad data & $\begin{array}{l}\text { Flag } 3 \text { data are suspect data in which unusual, and probably erroneous, features are } \\
\text { observed. }\end{array}$ \\
\hline Flag 4 & Bad data & Flag 4 data are data in which obviously erroneous values are observed. \\
\hline Flag 5 & Changed & $\begin{array}{l}\text { Flag } 5 \text { data have been altered by a QC center, with original values (before the change) } \\
\text { preserved in the history record of the profile. }\end{array}$ \\
\hline
\end{tabular}

changes or actions taken with respect to the profiles in that station. These history records are an integral part of the MEDS-ASCII format that allows retention of the original values if a value is changed. Each history record also contains a two-character code that identifies the action taken. For example, if a temperature spike is changed through interpolation, the original value is still present in a history record that also records the reason for the change: an "SP" code, in this case.

We also flag each temperature value with the data quality in accordance with the Global TemperatureSalinity Profile Program (GTSPP; Table 1). Generally data of quality 1, 2, or 5 are considered "good" without reservation for scientific use. Data of quality 3 or 4 are considered "bad" and rejected from all analyses. Data of quality 0 are used as if they were good.

We aim to eliminate bad data that might affect the end user and we reject only the part of a profile that is judged bad or questionable. This may mean we reject some good data because it is unclear whether it is good or bad, but we believe that retaining bad data in the archive would be more costly than the rejection of a small amount of good data. By looking at profiles in detail, in the context of their near neighbors in space and time, and eliminating as much bad data as possible, we aim to provide a database that will be useable for both large-scale and finescale ocean analyses.

In some cases, entire cruises were rejected though we did not explicitly use cruise information in the QC. Biased cruises or cruises with obvious location errors (e.g., tracking across land) were easily identified based on date and data-type information and eliminated.

We made no attempt to QC salinity. Various other groups who are more experienced with salinity (e.g., Hydrobase II) have performed this task with excellent results and it is outside the scope of QuOTA.

The retention of the flags and failure modes for a temperature profile is a critical part of the system: once flagged, any feature or fault does not need to be reassessed on subsequent rounds of QC. We can also tally these codes and use them to assess the effectiveness of the QC process and which failure modes are most common.

To make sure that we tracked data quality through all the contributing datasets, we assigned a priority level to each data source. CSIRO SOOP and WOCE were considered the best quality data since each profile had been subjected to the highest level of scientific quality control (manual examination by an expert). Other datasets were assigned lower levels of priority depending on our perception of the QC they had been subjected to. This assessment of input quality allows us to retain the data of highest quality when choosing between identified duplicates in the next step.

\section{b. Step 2: Elimination of duplicate profiles}

The source datasets are highly overlapping so a process was required to eliminate duplicate profiles and keep only the highest-quality copy. Besides the scientific imperative to remove these duplicates, we risk biasing the statistical parameter estimates to be used in subsequent screening procedures. Also, if duplicates are not identified and removed we would process each profile multiple times and so unnecessarily increase the processing time and work load.

Checking datasets for duplication is not as simple as it sounds. Stations that are identical may have errors in location by more than $30^{\circ}$ latitude or longitude or both. Some are placed in the wrong hemisphere. Two temperature profiles may represent the same data but be of different resolution or precision. It is impossible to detect and eliminate all duplicates, but we believe that the technique detailed below identifies most. 


\section{1) Exact duplicates}

Before combining data from different sources, we run two duplicate checking programs. First, we run a preliminary check to look for exact duplicates throughout a single database. By exact, we mean profiles where the depth/temperature measurements and the number of measurements are exactly the same. We ignore isothermal profiles and profiles with less than three points, which are more likely to give spurious matches. We accept two profiles as exact duplicates if more than $95 \%$ of the data pairs match; in practice, $100 \%$ of the data pairs usually match.

For each profile, we calculate the number of depths, the sum of the depths, and the sum of the temperatures. We then compare the summed temperatures and depths for all profiles with the same number of data points. If they match exactly, a depth-by-depth and temperature-by-temperature comparison is made. If $95 \%$ of the pairs match, we mark one or both as duplicates and remove them from the database. If the two profiles are close in space and time, the first is arbitrarily kept; if not, then both are rejected.

When the identified duplicates are manually inspected we find $100 \%$ of the profiles rejected were exact duplicates of profiles that were retained. Using this method we have found profiles $40^{\circ}$ apart in latitude and $30^{\circ}$ apart in longitude that are identical. This program was run over the worldwide WOA 2001 database and found that at least $0.66 \%$ of the database were exact copies of other data in the database. Once these have been removed from the database, we perform a second duplicate check looking for more subtle problems.

\section{2) NeAr duplicates}

Since profiles of different resolution or precision will pass the first duplicate test, we must now find profiles that are equivalent, but where one version has a lower precision. We term these "near duplicates." Because of the limitations of computing power, we only compare profiles near in space.

Each profile to be added to the archive is compared to all other profiles within $0.1^{\circ}$ latitude and longitude. Exact matches of data type (XBT/MBT/bathy, etc.), date-time, and position are considered true duplicate profiles without further comparison and only one copy (the one considered highest quality or highest resolution) is kept. Profiles of the same or similar data types (e.g., XBT and bathy copies of the same profile) that are close in space where more than $75 \%$ of their temperatures match are also considered identical when different data precisions are taken into account. We use both truncation and rounding of both temperature and depth to mimic changes that can occur as data moves through different computer systems.

If two profiles are near duplicates, we then check for several other errors that have appeared in the database. Reporting a station with local time instead of UTC is a common error: the profile with UTC time is kept. We have also found identical temperature profiles reported as much as $8 \mathrm{yr}$ apart with all other metadata identical. In this case, both stations must be rejected since year is a critical factor in calculations of climate variability and change; unless we have more information, we cannot choose between the two possibilities with confidence.

The station containing the profile from the highestquality source is generally kept and the other rejected unless the latter is of higher vertical resolution. In this case, we accept that the profile will need closer scrutiny than if we had kept the profile from the higher-quality source.

Identified duplicates are written to a separate database so we can check how effective the process is. When 2000 of these were checked by hand, only 26 of the identified duplicates were not true duplicates, giving confidence that our algorithms identify near-duplicates effectively. Most of the misidentified were cases of two instruments being deployed simultaneously (e.g., XBT dropped with a CTD giving a similar profile) or two profiles very close in space and time in an area where properties change very slowly. Since one profile was retained in the database, we consider this error level acceptable. We now check only a small sample of the duplicates to ensure that the routines are working equally well in different ocean basins.

Exact duplicates that are from different years are written to a separate database. In this case, though few such profiles were found, only a small portion of these $(13.8 \%)$ were really duplicates; the rest were returned to the database. Because there are few, checking them by hand is recommended. The bias from having duplicate profiles far apart in time makes this process worth the effort required to eliminate them from the database.

Since the WOA 2001 and NODC GTSPP databases are highly overlapping, the number of duplicates is large. Even within these databases, however, we typically find about $6 \%-10 \%$ of the data are duplicates.

After duplicate checking we have a nearly unique set of temperature profiles that are then checked using a statistical screening protocol for quality control.

\section{c. Step 3: Statistical screening}

Use of statistics to find unusual or suspect profiles has long been the primary means of quality controlling large historical data archives. Often this is the only 


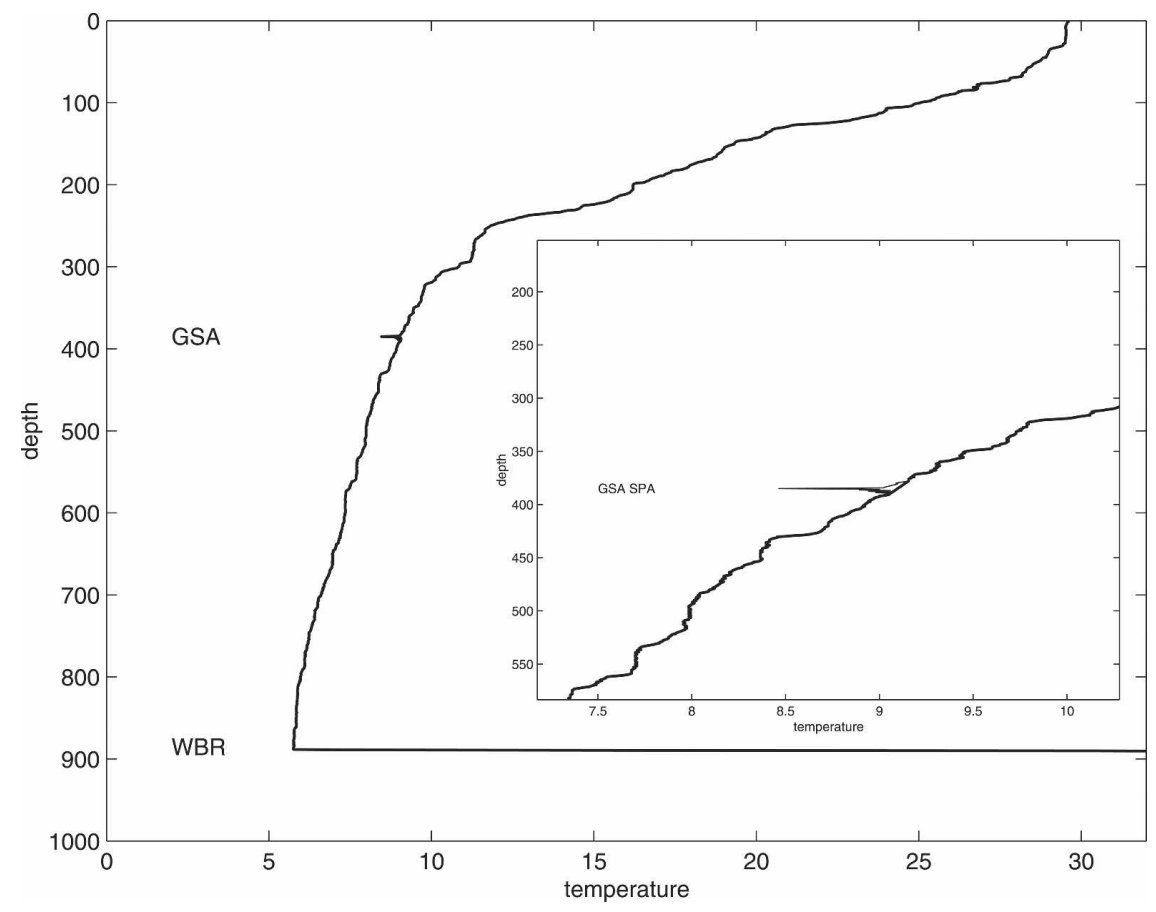

FIG. 1. A series of small but spurious spikes detected in the gradient checking steps. These spikes were removed through interpolation without affecting the underlying profile as seen in the insert, which is a close-up of the spikes with the interpolation results. The gradient routine also automatically flagged the wire break at the bottom of this profile, saving time during the hand QC.

method used to identify bad data. QuOTA examines the statistics of many parameters associated with ocean temperature profiles, strives to form the "best" available statistics of these parameters (i.e., based on a "good" master dataset), and iterates this process to improve the outcome. We also found a very simple test to be quite powerful in identifying bad data-looking for anomalous vertical gradients.

\section{1) STEP 3.1: GRADIENT CHECK}

The first screening step is to check all profiles for anomalous vertical gradients of temperature, which experience has shown often results from instrument failure. We find many faults through this test, especially for the tropical and subtropical parts of the archive where temperature as a function of depth, that is, $T(z)$, is well behaved.

Gradients are defined as

$$
[z(i z+1)-z(i z)] /[T(i z+1)-T(i z)],
$$

where $i z$ is the depth index in the profile, $z$ is depth, and $T$ is temperature. Forming the gradient as a slope gives a more tightly constrained value, particularly where temperature inversions are found, which is easier to screen than the more usual inverse of this formula. If temperature is isothermal, then the gradient is effectively infinite and so passes this test. Any gradient found between -0.4 and $12.5 \mathrm{~m}^{\circ} \mathrm{C}^{-1}$ is flagged as suspicious.

We also automatically deal with a known problem in XBT data-the so-called surface transients introduced into the data because of the thermal lag in response time of the instrument. Temperatures shallower than $3.6 \mathrm{~m}$ are flagged and replaced with missing values (Roemmich and Cornuelle 1987; Bailey et al. 1989). Wire breaks, where temperatures are greater than $36^{\circ} \mathrm{C}$ or less than $-2.8^{\circ} \mathrm{C}$ at the bottom of the profile, are automatically flagged and rejected, saving time when manually checking the data. Large single point spikes $\left(>1^{\circ} \mathrm{C}\right)$ are found and removed from the profile using interpolation for high-resolution profiles (e.g., Fig. 1) and replacement with a missing value if the profile is of low vertical resolution (e.g., Fig. 2). Smaller spikes remain to be removed by the manual QC. Each profile that is modified or labeled suspicious is manually examined to determine whether the gradient has caught an instrument failure or a real feature of the ocean. Failures are flagged and rejected from the final dataset.

Gradient checking routines are very effective in iden- 


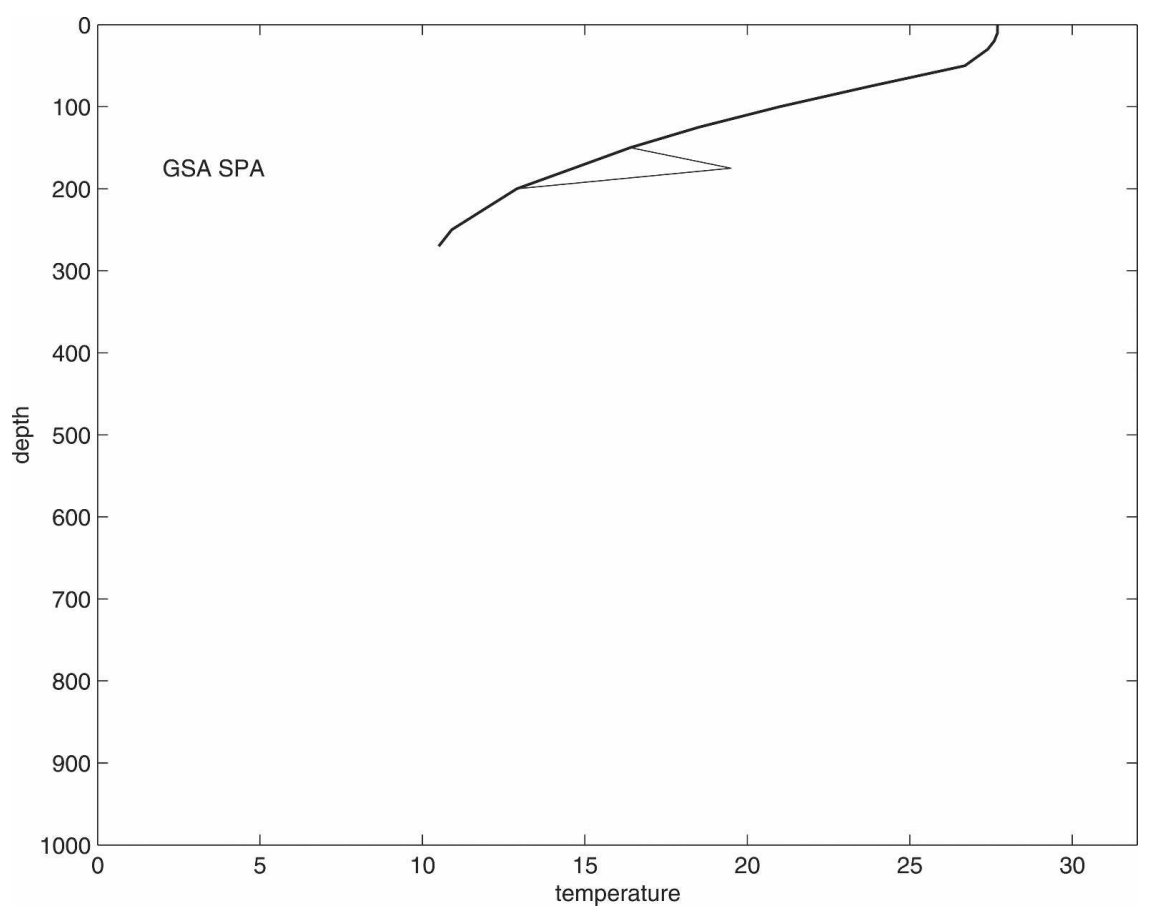

FIG. 2. Automatic interpolation of single spikes saved significant time during the hand QC of gradient failures. One data source in particular showed spikes in an area where no other data showed inversions (shown above). The spike was replaced with a missing value because this was a low-resolution profile (the results seen as a straight profile).

tifying bad data. While only $9 \%$ of the total archive failed this test, $60 \%$ of these profiles contained bad data as identified by manual inspection, and this represented $35 \%$ of all the bad data ultimately identified in the dataset. The remaining bad data are either subtle (temperature offsets that do not exhibit an unreasonable gradient) or small spikes below the threshold with a temperature error of less than $0.2^{\circ} \mathrm{C}$.

One of the most powerful features of the gradient test was its effectiveness in detecting the depth at which an XBT hits the ocean bottom. This often results in a small spike in the data, which was flagged by the program. Some previously unrecognized bottom hits were detected in data that had already passed our most stringent scientific QC. For example, Fig. 3 shows a small spike resulting from a hit-bottom event that would have passed all other tests.

The efficiency of this test varies with ocean region. In the Southern Ocean where temperature exhibits a lot of finescale variability, more profiles fail the gradient test and must be checked manually. Profiles that contain only good data failed because of the large inversions that are typical of this region. We are also finding that a larger number of profiles must be checked near the Red Sea and Persian Gulf outflows for similar reasons.

\section{2) STEP 3.2: Statistical PARAMETER SCREENING}

Originally our intention was to form averages and standard deviations of various parameters from a highquality "clean" master dataset and use these to look for outlier profiles in the candidate dataset. A profile would be flagged as "suspect" when one of its parameters lay outside the climatological distribution by some multiple of the standard deviation. However, for the eastern Indian Ocean, we found that most of the region had too few profiles in our master dataset (WOCE and CSIRO SOOP archive) to form reliable statistics. Thus an iterative process is used, in which a climatology of means and standard deviations of parameters is first formed based on the clean master dataset if sufficient data exist, supplemented by data from the candidate dataset if necessary. A gross screening is then executed using weak criteria to identify the worst of the data errors, manual QC is performed, and the process iterated a second time with stricter tests using statistics recalculated from the entire, combined dataset (master plus candidate, good data only). In ocean regions where large amounts of high-quality data exist from modern programs such as WOCE or Argo, our initial approach is becoming more feasible. 


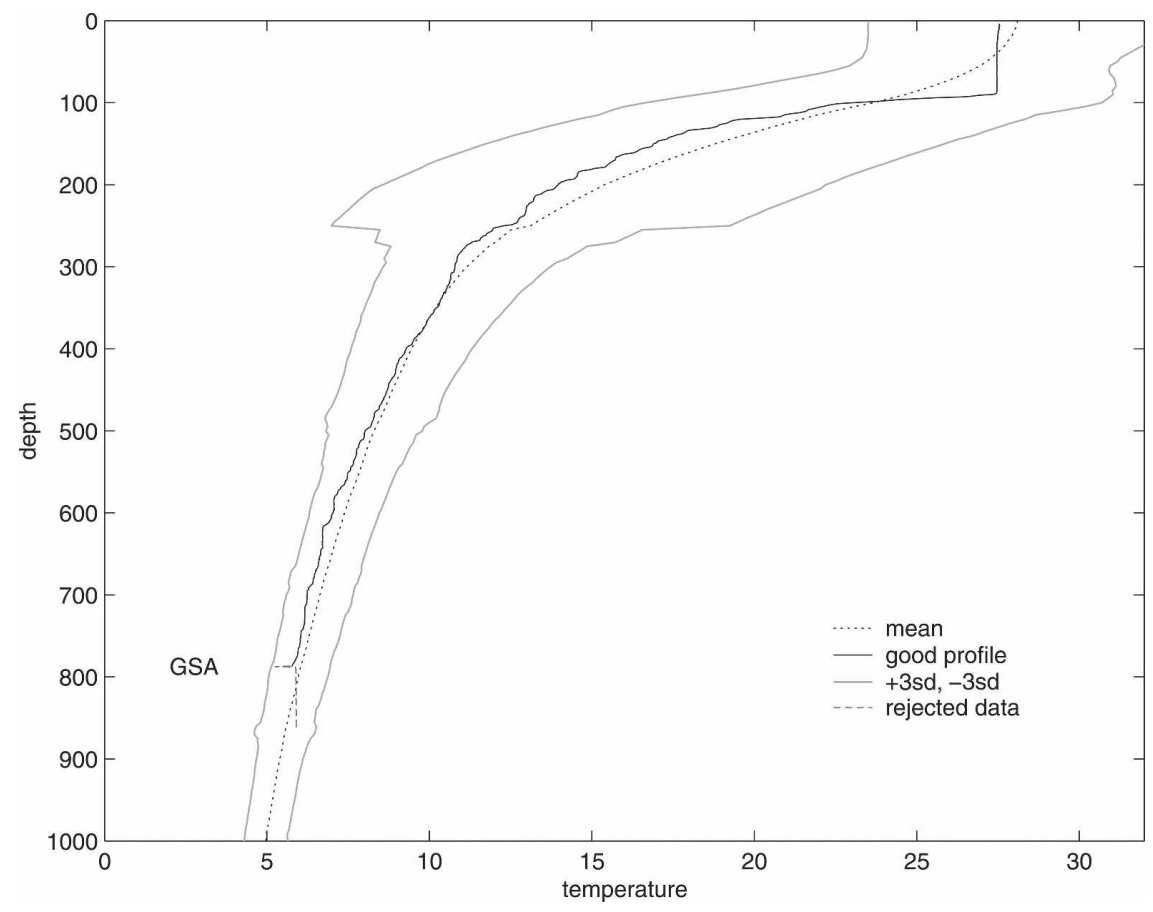

FIG. 3. The small spike marked by the gradient-small-accept (GSA) flag was an artifact of this XBT hitting the ocean bottom; the data below the spike do not differ from the statistical background sufficiently to be detected using the statistical screening programs, but is clearly not part of the profile and was identified by our gradient checking methods.

We use three types of parameters (Table 2): those that form a single value for each profile, those that are interpolated by depth (using 5-m depth bins), and those that are interpolated by temperature (using $1^{\circ}$ temperature bins). Parameters following temperature surfaces allow us to exploit the fact that much upper-ocean variability is in the low vertical modes and thus largely conserves the thermocline shape.

Much of the data in the archive is of low vertical resolution and was formed by selecting and storing only

TABLE 2. Parameter definitions and abbreviations. Temperatures were binned by $1^{\circ}$; depths were binned by $5 \mathrm{~m}$ where applicable. All integrations used the trapezoidal rule.

\begin{tabular}{|c|c|c|c|}
\hline Parameter & Abbreviation & Grid type & Description \\
\hline Sea surface temperature & SST & None & $T$ at the shallowest $z$ if $<$ than $10 \mathrm{~m}$ \\
\hline Mixed layer depth & MLD1 & None & $z$ where the $T$ is more than $0.5^{\circ}$ cooler than the SST \\
\hline Mixed layer depth & MLD2 & None & $\begin{array}{l}z \text { where the gradient change (absolute change of } T / \text { change of } \\
z \text { ) is greater than } 0.25^{\circ} \mathrm{C} \mathrm{m}^{-1}\end{array}$ \\
\hline$T$ at $100 \mathrm{~m}$ & $\mathrm{~T} 100$ & None & \\
\hline$T$ at $250 \mathrm{~m}$ & $\mathrm{~T} 250$ & None & \\
\hline$T$ of a $z$ surface & $T(z)$ & $z$ & $\begin{array}{l}\text { Estimated } T \text { at } z \text { calculated by linearly interpolating between } \\
\text { the } T \mathrm{~s} \text { at the depths closest to the depth } z\end{array}$ \\
\hline$z$ of a $T$ surface & $z(T)$ & $T$ & Estimated $z$ at $T-$ opposite of above \\
\hline$T$ gradient at $z$ & $d T d z$ & $z$ & $\begin{array}{l}\text { The gradient is calculated using the temperatures of the depths } \\
\text { bracketing } z \text { (similar to the gradient check already } \\
\text { performed) }\end{array}$ \\
\hline$z$ gradient at $T$ & $d z d T$ & $T$ & As above, but calculated over $T$ bins \\
\hline Cumulative integrated raw $T$ & IntRaw & $z$ & $\begin{array}{l}\text { Integral from surface to depth of } T d z \text {, where } T=\text { observed } \\
\text { temperature }\end{array}$ \\
\hline Cumulative integrated bin $T$ & IntBin & $z$ & $\begin{array}{l}\text { Integral from surface to a (bin) depth of } T d z \text {, where } T= \\
\text { binned temperature }\end{array}$ \\
\hline Averaged integrated raw $T$ & AvgRaw & $z$ & Integrated observed $T$, divided by the $z$ \\
\hline
\end{tabular}


inflection points in the profile. These include most MBT data, all real-time (BATHY) data from XBTs, and real-time (TESAC) data from CTDs as well as a significant proportion of the data from early XBTs. Though of low resolution, the data still accurately represent the underlying profile. To ensure that we had a "full" profile before we calculated our parameters, we reconstructed the underlying profile by interpolating to a 2-m vertical grid. Data that did not contain information about the underlying profile structure, such as bottle data, were not interpolated.

\section{(i) Forming the statistics}

Our approach is based on regional differences in ocean characteristics, rather than tracking decadal variability. While statistical means based on time would be better given that the ocean is not stationary, in practice this would severely limit when and where statistics could be formed because of the uneven distribution of data through time. We considered that regional differences were more important than decadal differences and chose to create our statistics from smaller boxes rather than smaller time periods.

For each parameter and where enough qualitycontrolled data are available in a geographical box of $5^{\circ}$ latitude $\times 10^{\circ}$ longitude, parameter averages and standard deviations are calculated based on the master dataset. If there were fewer than 100 profiles, we expanded the box boundaries. First we doubled the longitudinal borders and, if there were still too few profiles, we then expanded the latitudinal boundaries. Finally, if there were simply not enough profiles in this expanded area, the un-QCed data in the candidate set were included to produce the statistics.

Even if there were very few observations in a box, we calculated and stored a mean and standard deviation along with the number of profiles that contributed to the statistics. We could therefore decide later whether to use these numbers and in some cases, where these statistics looked reasonable in areas where the ocean structure was more stable, we screened against means from as few as 20 profiles. Though this meant we sometimes had to look at data that was good because it failed a less robust statistic, we did not find that we missed bad data because of this methodology.

Profiles in areas where statistics could not be calculated were checked by the QC operator. All profiles were therefore checked either by an operator or by statistical screening.

If expanded boundaries were used to calculate the background statistics, we screened the parameters based on the original boundaries, not the expanded boundaries so, if a profile occurred in two statistical calculations through the expansion of a neighboring box, it was screened only against the statistics of its "home" box. Both parameters (for each profile) and statistics (boxed by latitude and longitude) are stored in netCDF files, which can be used for other purposes.

All subsequent iterations use only the "good" dataset for calculation of the statistics, on the assumption that the worst of the data errors have been eliminated and the statistics are robust after manual QC of all initial failures.

\section{(ii) Determination of critical values for screening}

The critical value $(\mathrm{CV})$ is the number of standard deviations from the mean outside of which a parameter would be judged to have failed a test. Chosen critical values varied depending on the characteristics of the parameter. Some were normally distributed; others were not (Fig. 4). As one would expect, parameter distributions were also dependent on the quality of the means and standard deviations used to normalize them (Fig. 4).

Typically, bad data are not evenly distributed around the mean. Most gross instrument failures, particularly for XBTs, which form a large part of the more modern database, result in warmer temperatures [see leakage and insulation penetration (Bailey et al. 1994)]. Sometimes all data from a voyage showed large offsets, either cold or warm. Large numbers of MBTs had to be rejected because of these sorts of systematic offsets due either to calibration/digitization errors or perhaps because of position errors.

These can bias means, which subsequently cause good data to fail the tests and bad data to pass. Cumulative integrated binned temperature in particular is subject to these bias errors, and the statistics from the mixed-quality dataset are so biased as to make this parameter useless in the first screening iteration. See Fig. 5 for the critical values used in the initial screening for each parameter as well as how well each parameter performed in catching bad data and passing good data. After screening, we then visually inspected every profile flagged.

In addition, all profiles from the months of January through March plus June were fully manually qualitycontrolled to produce a dataset that we could use to evaluate our screening process. We could thus assess the performance of our semiautomated method when we changed the critical values, comparing what was caught by the statistics to what is "best"-visual inspection of every profile by an expert.

Initially, since all bad data are known for the master dataset, we used it to compare the number of profiles with known bad data caught by a given $\mathrm{CV}$ for a given 


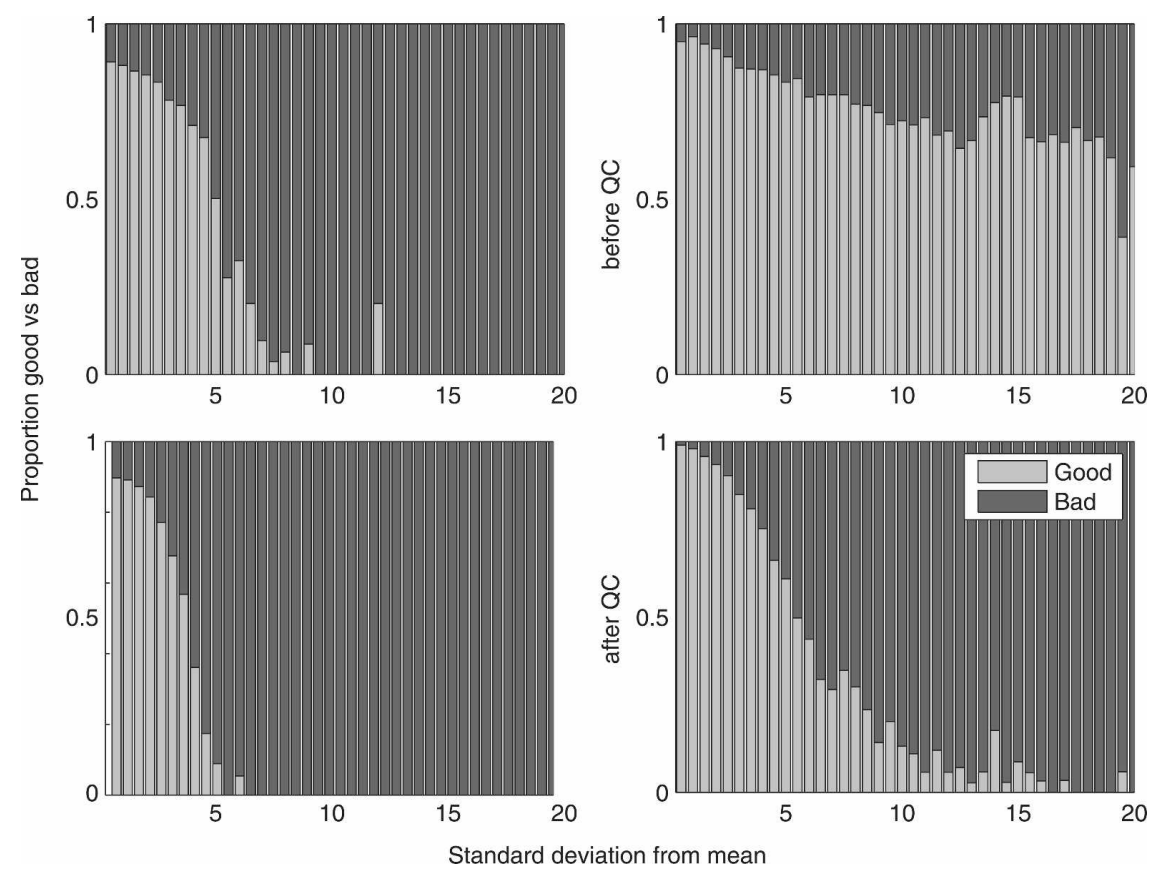

FIG. 4. Fractions of known good data as a function of std dev around two climatologies for two parameters: (left) T100 and (right) IntBin on the right (see Table 2 for definitions). (top) The means and std devs were based on data of mixed quality and (bottom) the means and std devs were based only on good data, resulting in better means and tighter std devs. The improvement of the behavior of the statistics is noticeable - bad data in the reference climatology used to generate the means and std devs produce a broader deviation spread and nonnormal distributions in the top.

parameter to the number of profiles caught without bad data. The point at which the number of good profiles caught increased significantly without a comparable increase in the number of bad profiles caught was chosen as the $\mathrm{CV}$ for screening the dataset we wished to add. Plots of the number of good and bad profiles caught by an increasing $\mathrm{CV}$ were visually examined and the screening $\mathrm{CV}$ was chosen for each parameter. A more sophisticated test would be to allow CV to vary geographically but this was not attempted here.

For the parameter T250 (temperature at $250 \mathrm{~m}$ ), for example (Fig. 6), a CV of 3 or 3.5 catches a large proportion of the bad data but the proportion of the good data caught is still low. We were thus able to tailor the critical value for each parameter and for each screening iteration to reflect the distribution of the good and bad data about the mean.

This process was performed both for the first screening and for the final screening and the resulting CVs used are shown on the $x$-axis labels of Figs. 5 and 7.

\section{(iii) Screening}

Not unexpectedly, we found that some parameters were much better at discriminating between good and bad data (Fig. 5). We also found that some statistics based on the candidate set were so biased by bad data that they were useless for screening-particularly when there were few observations in a box and we had been forced to use all of the data (including that from lowerquality sources) to calculate our statistics. This highlights the importance of building a high-quality "master" dataset and using it where possible to generate the statistics against which candidate data are screened.

After profiles flagged by the first statistical screening were visually inspected and quality controlled, the distribution of the data around the means tightened and the reliability of the statistics improved as the worst of the data were removed. We then recalculated the means and standard deviations and the data were rescreened using tighter critical values (see Fig. 7). Profiles that had passed the first test and then failed this second test were visually inspected.

Using smaller CVs and a better climatology we found more subtle errors in the data, and in fact found some bad data that had slipped through the expert quality control applied to the WOCE archive. The effect of better means and standard deviations can best be seen in the comparison between MLD1 in the first screening 


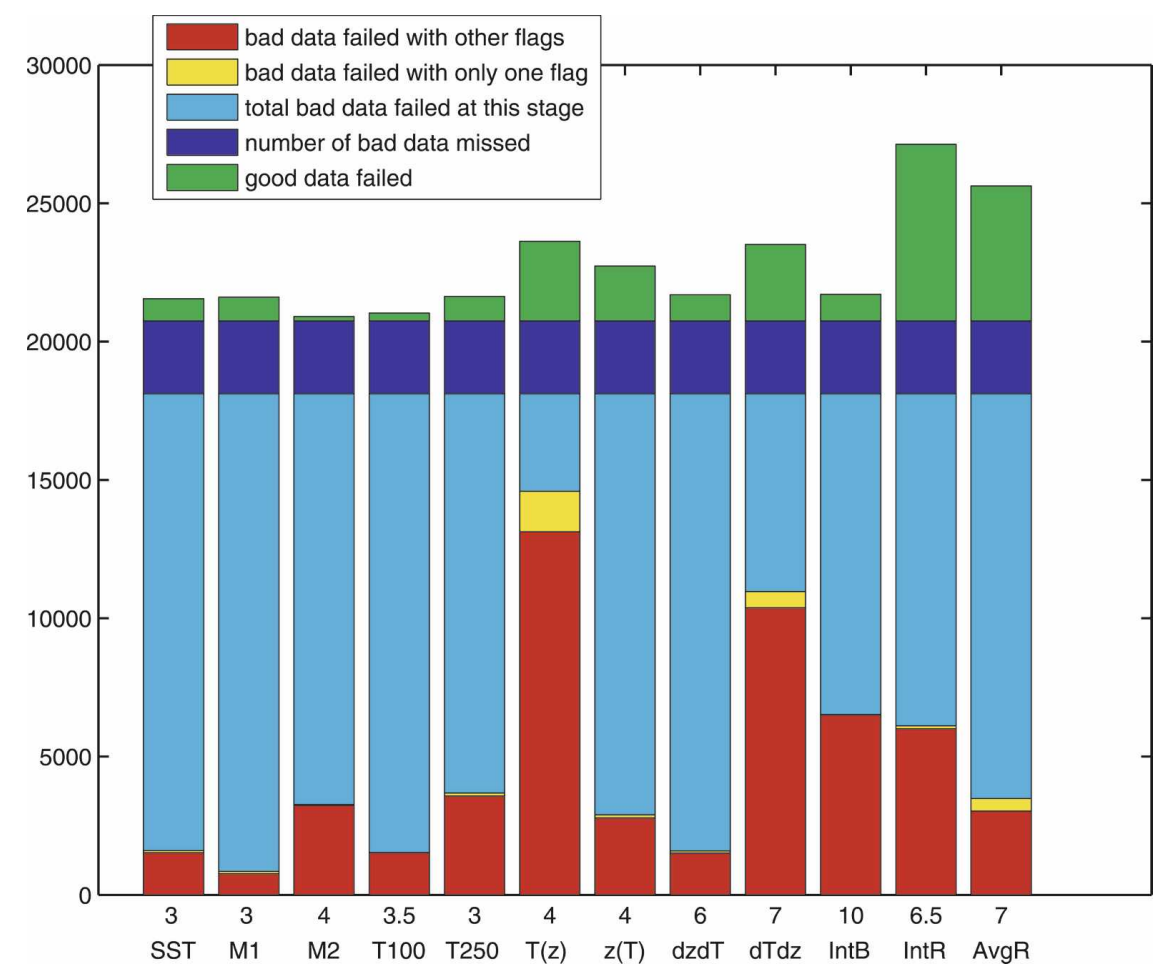

FIG. 5. The number of profiles caught by various parameters in the initial screening using the CVs marked along the abscissa. The top of the blue bar indicates the total number of bad profiles known to be in the database. The top of the aqua bar shows the total bad profiles caught at this screening stage by all parameters. Red bar shows the number of bad profiles caught by that parameter (jointly with the others), while the yellow component shows bad profiles caught by only that parameter. The green portion shows the number of good data caught by the screening process. Hence, the process overwhelmingly flags bad data and relatively little good data.

and MLD1 in the second. The same CV was used in both cases but more bad data were caught in the second screening and fewer good data were also caught. Several of the parameters also caught fewer good data when better means were used, despite smaller CVs. Though these differences are small, this shows how important good background statistics are to this process and how bad data in your master archive can bias the statistics.

Reducing the CVs for each parameter will catch a higher proportion of the bad data, but the cost is that more good data must be examined, which is expensive (e.g., see Fig. 6). Depending on the size of your dataset, reducing the CVs might be tolerable, but for very large datasets (there are seven million profiles globally at present), overflagging good data incurs a serious cost.

We also determined which parameters were most effective in the screening process. In practice, some parameters are much better at discriminating between good and bad data. For this analysis, we screened using all parameters and looked at the failures. By eliminat- ing parameters that flagged unreasonable numbers of good profiles (IntRAW and IntBin) we found that we flagged 2000 fewer profiles, yet missed only 38 profiles that had bad data. The final result is shown in Table 3 . This can save time when looking at very large datasets. The assumption here is that the profiles missed have minor, insignificant errors. This will be discussed later.

During visual inspection and manual QC other errors were also taken care of-data on land were rejected (our software does not automatically check for these at present but this can easily be added) and some deep profiles in shallow areas of the ocean were rejected as having severe position errors. We plan to add the ability to compare profile depth to ocean topography in a new version of the software.

In all comparisons, the parameter $T(z)$ performed the best, catching a significant proportion of the bad data and a low proportion of the good data. The parameters $d T d z$ and AvgRaw also were effective. Between them, these three parameters caught $95 \%$ of the bad data that were caught by no other parameter. Yet 


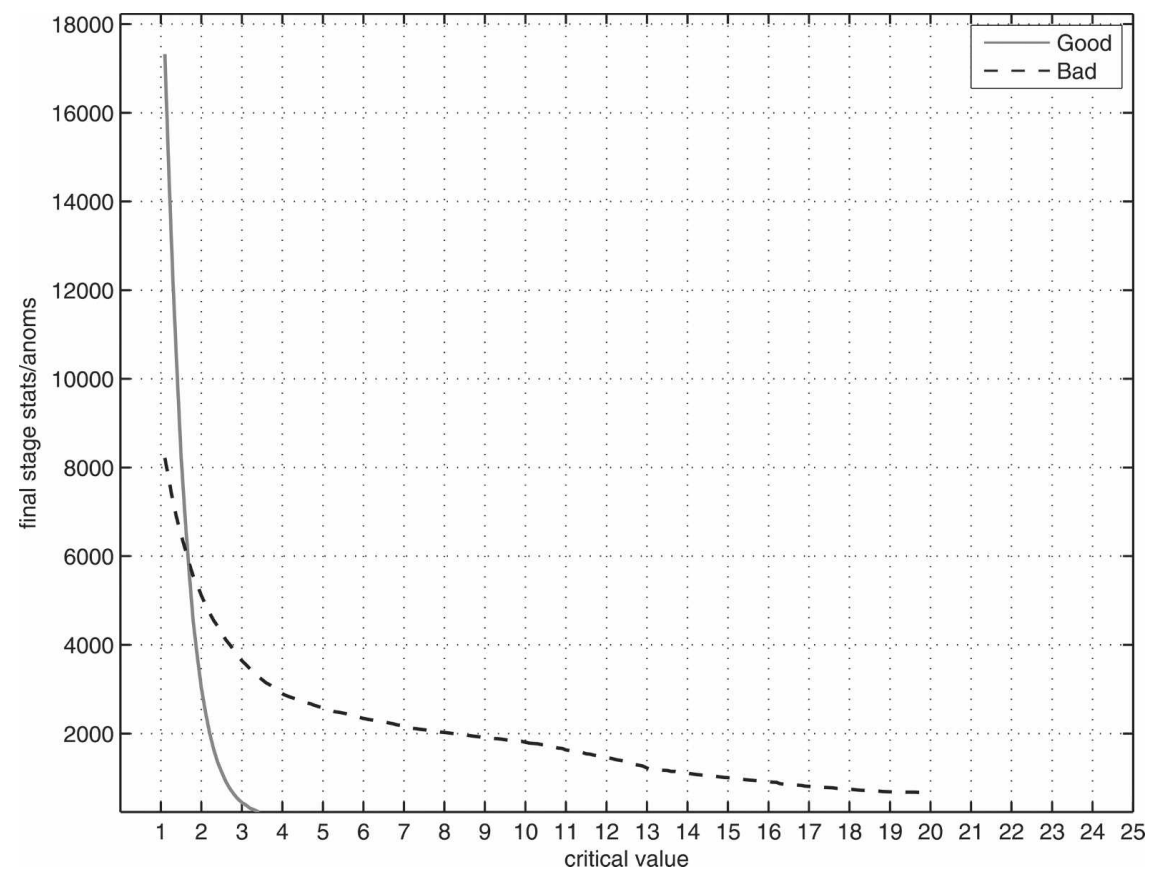

FIG. 6. Number of good (solid line) and bad observations (broken line) of temperature at $250 \mathrm{~m}$ vs std devs from the mean.

all parameters were responsible for catching at least some of the bad data that would otherwise have slipped through the statistical check, had that parameter not been included. Parameter $d z d T$, on the other hand, performed very poorly, catching a large percentage of the profiles with good data. This is most likely because of the problems calculating this parameter in areas of inversions where temperature is not necessarily decreasing with depth.

\section{d. A comparison with other methods}

In the eastern Indian Ocean, we found a total of 127161 unique stations. Of these, we know that at least 20749 contained bad data (identified during various QC processes, including QuOTA) or about $16 \%$ of the temperature profiles in the archive. QuOTA identified 19604 of these profiles during screening. It is not possible to catch every erroneous data value. This was evident when we tested QuOTA for the months of January-March and June, which were fully manually checked during the development of the software. Some bad data that are clearly due to instrument failures lie well within the climatology, while others occur in areas where there is little or no data with which to compare them and assessing data quality is very difficult. In areas of high variability or complicated ocean structure, the standard deviations might be naturally large, masking erroneous temperatures. At the end of our process, however, we feel that most, if not all, of the bad data have been eliminated and any remaining errors are so small that they will not affect most uses. Overall, for the months fully manually quality controlled, the accumulation of our tests caught $95.5 \%$ of the profiles containing bad data, leaving only $0.89 \%$ of the archive with (minor) bad data after passing through the QuOTA system. This is a significant improvement over our starting point where $16 \%$ of the profiles in the archive originally contained bad data.

One obvious "cost" of our approach is the need to manually check a significant proportion of the archive. The QuOTA procedures caught $17 \%$ of the profiles unnecessarily, requiring a total of about $32 \%$ of the dataset to be examined manually. It may be possible to tailor the statistical screening to allow for areas where extreme values are common (e.g., the Southern Ocean). Unfortunately, these areas are also the areas where the instruments fail more often. Checking data in these areas is relatively quick for an experienced operator and is probably worth the time to ensure that the maximum amount of bad data is eliminated.

To compare QuOTA with other methods, we looked at four quality control procedures (including doing nothing further to the data) and compared the resulting data quality with the time and effort required to achieve that outcome. Only the four months handchecked were used so we were confident we knew how 


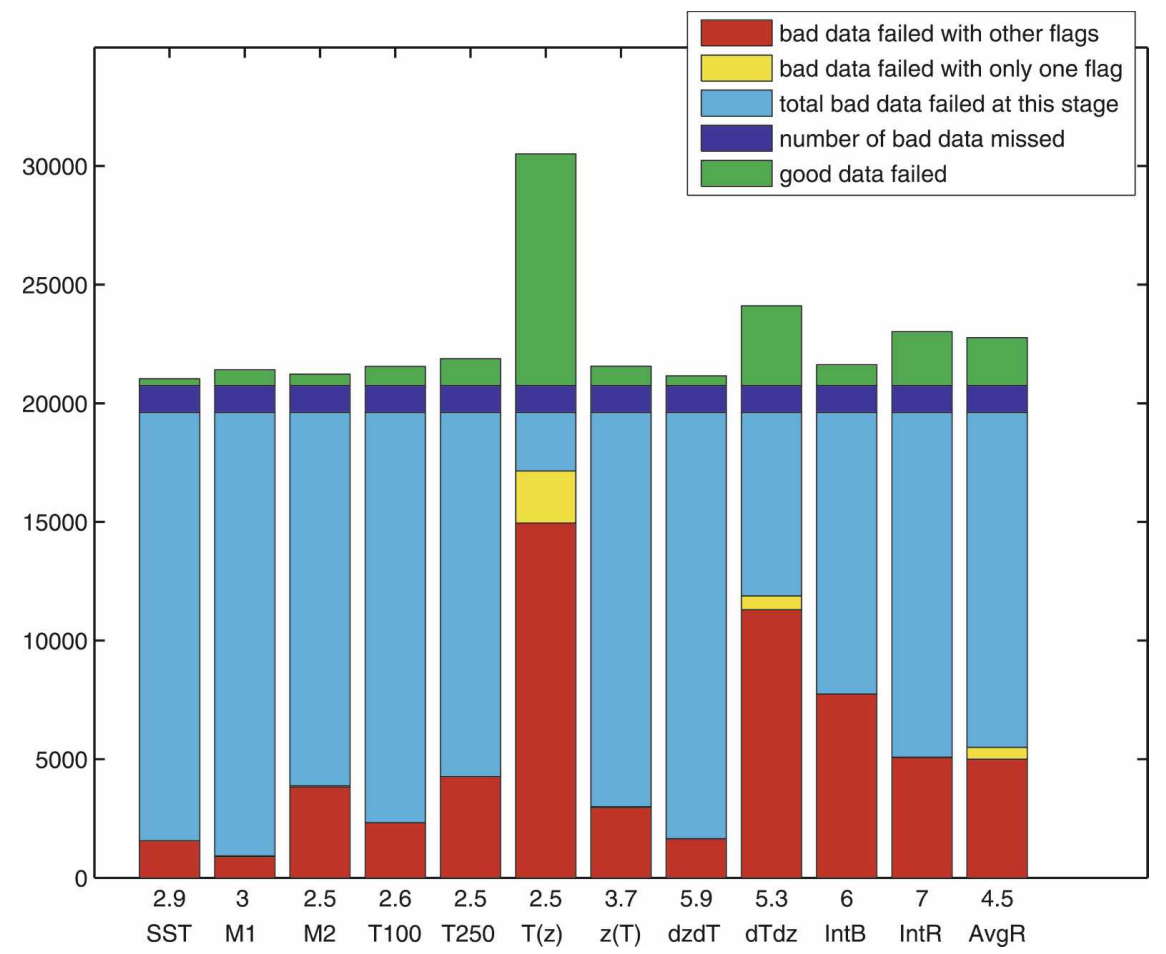

FIG. 7. Effectiveness of various parameters in flagging bad data in the final screening using the CVs on the $x$ axis. Legend as in Fig. 5.

much bad data existed independent of the methods being assessed. There were a total of 63470 profiles in these four months.

The first process considered was to use the data as it was, accepting the QC flags set by whatever source agency had provided the data. This is referred to as the "Do Nothing" approach. The second process was to simulate a QC procedure that relies solely on statistics to decide the quality of the profiles and rejects all tem- perature on depth surfaces that deviate from the mean by more than three standard deviations-we refer to this as the "Three Sigma" approach. The third process was QuOTA with two iterations (as described above). The fourth process was to QC every profile by hand (the four months of data used in this comparison).

For simplicity, we tallied number of profiles rather than number of data points. Different methods act in different ways. Data in the Do Nothing category often

TABLE 3. Parameters ranked by the number of total profiles caught from the total archive. These were calculated cumulatively so if a profile failed the $T(z)$ test, it was not considered for any further tests. The actual number caught by each parameter is dependent on the chosen CV but the pattern is relatively stable. Two parameters have been eliminated-IntRaw and IntBin cumulatively caught an extra 2080 good profiles but caught only 38 bad profiles that were not caught by other parameters.

\begin{tabular}{lccccc}
\hline \hline Parameter & Rank & No. of good caught & No. of bad caught & Total caught by this parameter & Cumulative total caught \\
\hline$T(z)$ & 1 & 9756 & 17148 & 26904 & 26904 \\
$d T d z$ & 2 & 2123 & 786 & 2909 & 29813 \\
AvgRaw & 3 & 1742 & 634 & 2376 & 32189 \\
MLD1 & 4 & 335 & 43 & 378 & 32567 \\
MLD2 & 5 & 215 & 66 & 281 & 32848 \\
$z(T)$ & 6 & 182 & 27 & 209 & 33057 \\
$d z d T$ & 7 & 84 & 19 & 103 & 33160 \\
T250 & 8 & 1 & 1 & 2 & 33162 \\
SST & 9 & 0 & 1 & 1 & 33163 \\
T100 & 10 & 1438 & 18725 & 33163 & 0 \\
Totals & & & & & 0 \\
\hline
\end{tabular}




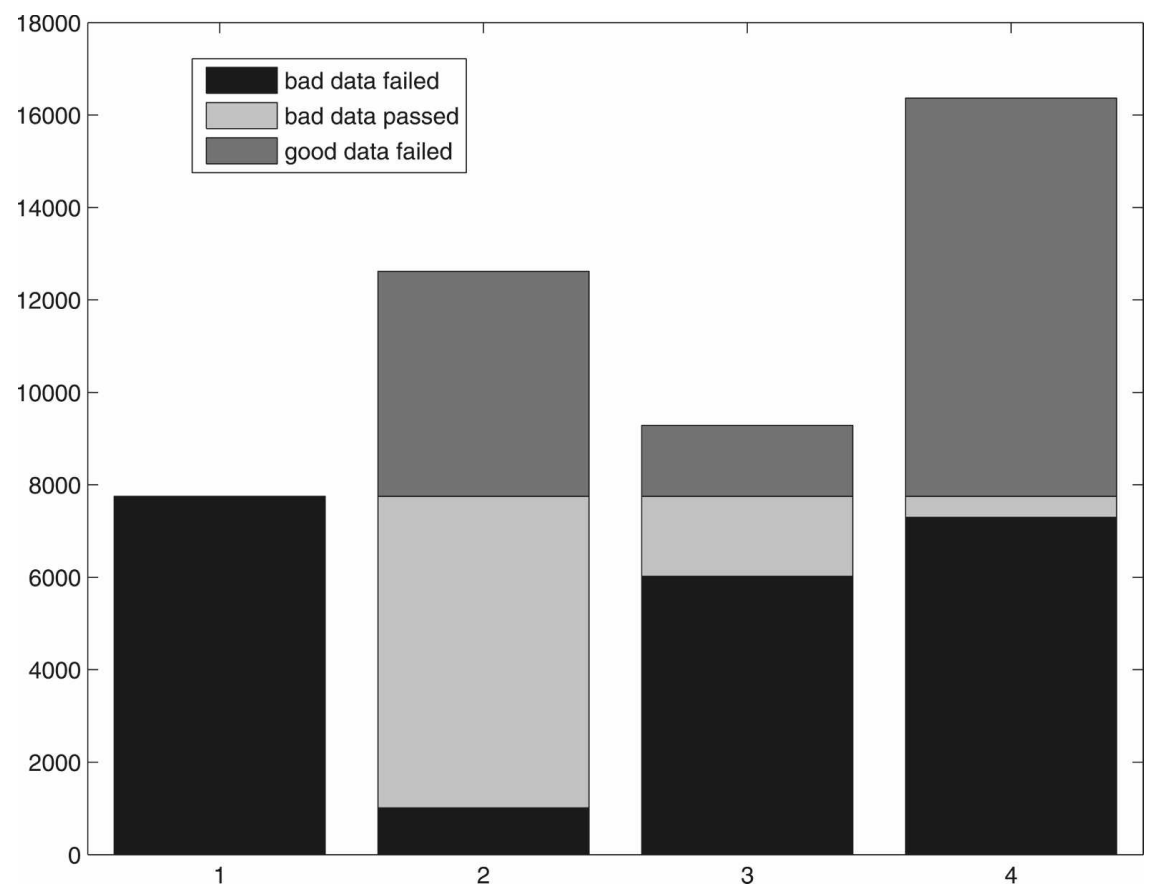

FIG. 8. Results of four different approaches to identifying bad data in the archive. The 4 months of fully QCed data were used in this analysis (63 470 profiles). Column 1: The number of known bad profiles in the 4 months of data inspected by individual examination of each profile. This represents the best approach. Column 2: The number of profiles in the same 4 months with bad data flagged (black bar) when the dataset was assembled; i.e., no QC has been done beyond what was present when the data were acquired. The lightest bar indicates the profiles with bad data missed and the dark gray bar indicates the good data that have been flagged as bad and would be lost in any analysis if this QC were accepted. Column 3: The numbers of profiles with bad data flagged in the same 4 months (shading as above) using the Three Sigma approach. The lightest bar indicates the bad data that would remain in the database using this method; the dark gray bar shows the number of good profiles rejected in error. Column 4: The results of the QuOTA system for the same 4 months after two screening iterations. The proportion of bad data missed is significantly smaller than with the other two procedures. Though a significant number of good data were caught, they were not rejected after hand-QC and so remain in the database.

had only a small part of the profile rejected, not identifying bad data that were caught by other methods or rejecting data that were well within climatologies and judged good in the QuOTA QC. Depending on how it is applied, the Three Sigma approach could either reject the profile below the point of deviation, reject the entire profile, or reject only those points that deviate from the mean. QuOTA generally rejects all data below a failure because our experience has been that such data are unreliable (with some exceptions).

The trade-offs in quality versus expert labor invested in the archive are illustrated in Fig. 8. Column 1 shows the total number of profiles containing bad data for the four fully-QCed months in the final high-quality archive. This represented $18.8 \%$ of the data subset. In the Do Nothing approach (column 2), a significant proportion of the profiles containing only good data are erro- neously identified as bad (dark gray) and there is also a large proportion of the profiles containing bad data that had either not been QCed or had been erroneously accepted as good (light gray). In the past, data centers stripped off any QC flags applied by the data originators and then ran their own QC processes over the data. These processes were designed to catch only the most major data errors. This situation is changing but has resulted in information being lost when data were submitted to international archives. In the Three Sigma approach fewer good data are rejected in error, but there are still a significant proportion of bad data remaining in the dataset (light gray-about 20\%). QuOTA with two screening iterations (column 4) captures nearly all of the bad data (black), leaving little missed $(4.5 \%$ of the total profiles with bad data remain, representing $0.89 \%$ of the profiles in this subset-light 
gray). The cost, however, is that it requires about $35 \%$ of the total dataset to be examined by hand, about $1 / 2$ of which are good data (dark gray bar). The real cost of this system is the expert time required to perform the data assembly and quality control. An expert can quality control about 500 profiles per day, suggesting a parttime operator could complete the Indian Ocean (around 400000 profiles) in two years.

\section{Summary and conclusions}

Complete manual quality control to a level useful for climate studies of the historical ocean temperature archives (as they are now assembled) is simply not affordable. Here we have described an approach to produce a historical temperature archive of high quality-that is, where as many of the profiles as possible are unique and have undergone a high standard of quality control. Compared to a purely automated system, the technique requires a large amount of time from quality control experts. The semiautomation of several of the steps (duplicate removal and scanning for suspect data) makes this task feasible, but compared to fully automated screening it avoids the loss of much good data and inclusion of significant amounts of bad data. Ocean basins with larger datasets will take longer, depending on how many experts are available to quality control the data. By building on the success of the Global Ocean Data Archeology and Rescue activities (Levitus et al. 1994) and utilizing datasets of known high quality, for example, those assembled under WOCE and those that are supplied by the Argo program (Koblinsky and Smith 2001), QuOTA makes it feasible to assemble the historical data and quality control it to a high level. As this would largely need to be done only once for the historical data of each ocean basin, the resource it would present for climate research would make it a worthwhile endeavor and the cost of keeping the archive up to date with new data would be within our reach.

Because of its high quality, QuOTA requires less averaging than previously available archives and should allow finer spatial resolutions to be achieved in climatologies. For example, the test region treated by QuOTA has been used to construct high-resolution regional seasonal climatologies and make interannual composites for the ocean of Western Australia (Feng et al. 2003) and the full basin version for mapping long term changes in temperature (Alory et al. 2007).

These data are freely available in gridded netCDF files from the first author (ann.thresher@csiro.au) and will shortly be put online for download. They are also incorporated in the CSIRO Atlas of Regional Seas, a high-resolution gridded climatology of the world's oceans (available, with documentation, through http:// www.marine.csiro.au/ dunn/cars2006/.experiments on the test data).

Acknowledgments. We would like to acknowledge the support of NOAA and the International Pacific Research Center in this work. We also thank Gary Meyers and Peter Hacker for their encouragement and Jeff Dunn and Rebecca Crowley for their advice and assistance.

\section{REFERENCES}

Alory, G., S. Wijffels, and G. Meyers, 2007: Observed temperature trends in the Indian Ocean over 1960-1999 and associated mechanisms. Geophys. Res. Lett., 34, L02606, doi:10.1029/2006GL028044.

Bailey, R., H. E. Phillips, and G. Meyers, 1989: Relevance to TOGA of systematic XBT errors. Proc. Western Pacific Int. Meeting and Workshop on TOGA COARE, Noumea, New Caledonia, Centre ORSTOM de Nomea, 775-784.

_ A. M. Gronell, H. Phillips, E. Tanner, and G. Meyers, 1994: Quality control cookbook for XBT data. CSIRO Marine Laboratories Rep. 221, 84 pp. [Available online at http:// www.marine.csiro.au/ gronell/cookbook/csiro.htm.]

Feng, M., G. Meyers, A. Pearce, and S. Wijffels, 2003: Annual and interannual variations of the Leeuwin Current at $32^{\circ} \mathrm{S}$. $J$. Geophys. Res., 108, 3355, doi:10.1029/2002JC001763.

Gronell, A. M., cited 2002: Scientific quality control of XBT data. [Available online at http://www.marine.csiro.au/ OceanObsNetworks/ScientificQualityControl.html.]

Koblinsky, C. J., and N. R. Smith, Eds., 2001: Observing the Oceans in the 21st Century. GODAE Project Office, Bureau of Meteorology, $604 \mathrm{pp}$.

Levitus, S., R. D. Gelfeld, T. Boyer, and D. Johnson, 1994: Results of the NODC and IOC oceanographic data archaeology and rescue projects: Report 1 . NODC Key to Oceanographic Records Documentation 19, NOAA/NESDIS, 73 pp.

- S. Sato, C. Maillard, N. Mikhailov, P. Caldwell, and H. Dooley, 2005: Building ocean profile-plankton databases for climate and ecosystem research. NOAA Tech. Rep. NESDIS 117, 29 pp.

Meyers, G., and L. Pigot, 2000: Analysis of frequently repeated XBT lines in the Indian Ocean. CSIRO Marine Laboratories Rep. 238, 43 pp.

Roemmich, D., and B. Cornuelle, 1987: Digitization and calibration of the expendable bathythermograph. Deep-Sea Res. I, 34, 299-307.

Stephens, C., J. I. Antonov, T. P. Boyer, M. E. Conkright, R. A. Locarnini, T. D. O'Brien, and H. E. Garcia, 2002: Temperature. Vol. 1, World Ocean Atlas 2001, NOAA Atlas NESDIS 49, 167 pp. 